\title{
EL INTERÉS PARTICULAR EN EL SISTEMA DE PARTIDOS POLÍTICOS EN COLOMBIA
}

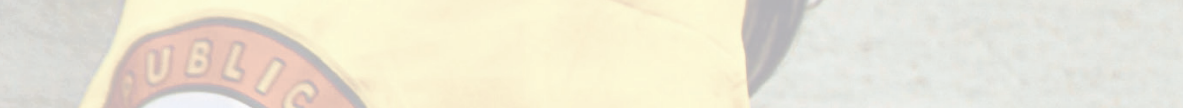

Belisario Velásquez Pinilla

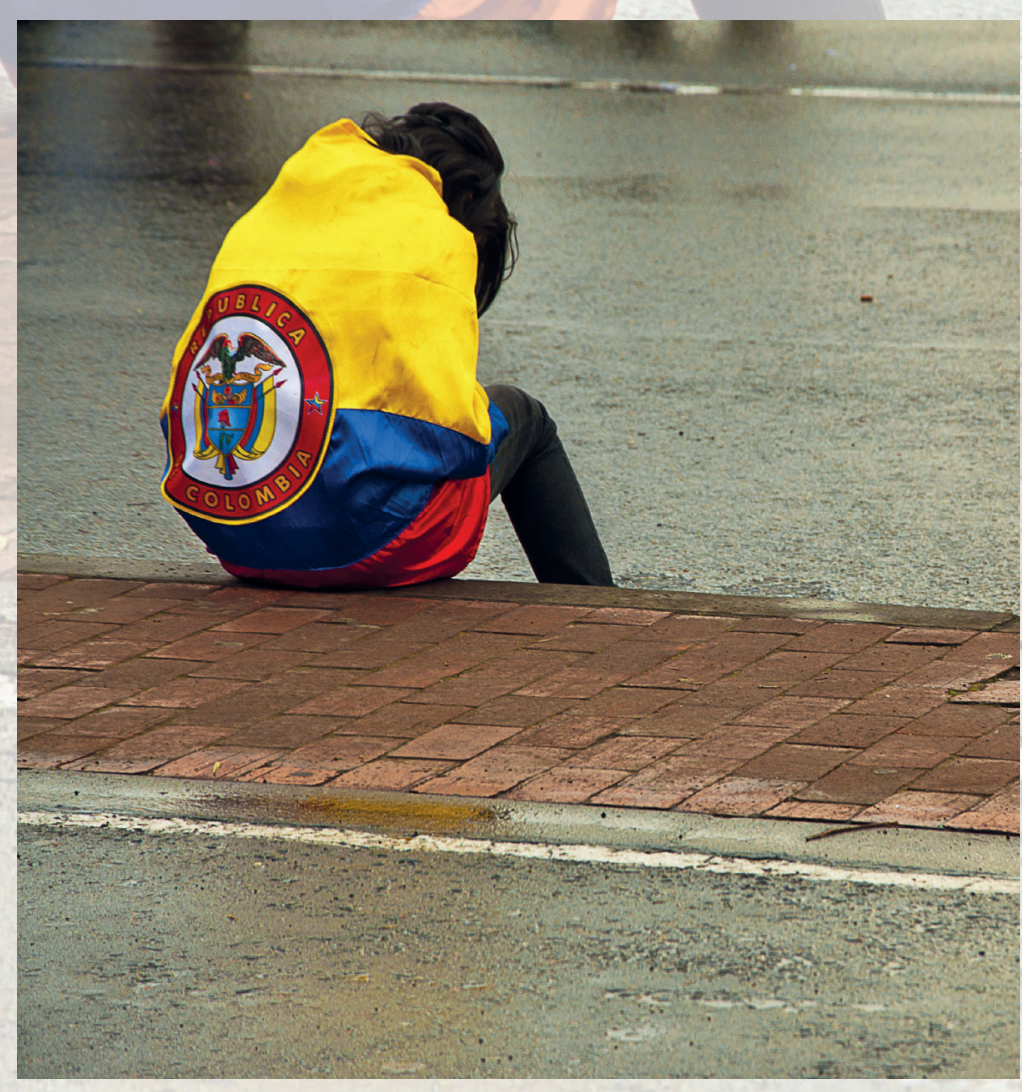

Título: Young woman wrapped in the Colombian flag sits on a sidewalk after a political rally in Bogota, Colombia on February 16, 2011 • Autor: Jess Kraft 



\title{
EL INTERÉS PARTICULAR EN EL SISTEMA DE PARTIDOS POLÍTICOS EN COLOMBIA
}

\author{
Belisario Velásquez Pinilla \\ BELISARIO SAS
}

\section{Resumen}

Uno de los principales problemas sociales que padece Colombia es la polarización ciudadana que producen las corrientes políticas en el momento de pretender establecer un modelo de gobierno. En el presente escrito se analiza el mal llamado "multipartidismo" desde una perspectiva histórica, y se intenta dilucidar la respuesta a por qué la partidocracia se ha originado en torno a intereses personales de los líderes políticos, en especial con ocasión del acuerdo de paz con las FARC-EP.

Palabras clave: partidos políticos, proceso de paz en Colombia, justicia transicional, partidocracia, transfuguismo, democracia.

El autor: docente investigador de la Corporación Universitaria Minuto de Dios, presidente de Belisario SAS. Correo electrónico: belisariol@belisario.com.co

Recibido: 30 de noviembre de 2017; evaluado: 5 de febrero de 2018; aceptado: 1 de marzo de 2018. 


\title{
PARTICULAR INTERESTS IN THE POLITICAL PARTY SYSTEM IN COLOMBIA
}

\author{
Belisario Velásquez Pinilla \\ BELISARIO SAS
}

\begin{abstract}
One of the main social problems currently faced by Colombia is citizen polarization produced by political movements that try to establish different models of government. This paper analyzes the so-called "multi-party system" from a historical perspective, and attempts to elucidate the answer to why "particracy" has originated around the personal interests of political leaders, especially on the occasion of the peace agreement with the FARC-EP.
\end{abstract}

Keywords: political parties, peace process in Colombia, transitional justice, particracy, party switching, democracy.

Author: Research professor at the Corporación Universitaria Minuto de Dios and president at Belisario SAS.Email: belisariol@belisario.com.co

Received: November 30, 2017; evaluated: February 5, 2018; accepted: March 1, 2018 


\section{Introducción}

Los partidos políticos en América Latina están provistos de una amplia diversidad, debido a la compleja estructura clasista y a la existencia de numerosos grupos y capas sociales intermedias. Las primeras asociaciones políticas surgieron en Europa en el siglo XIX como partidos de dos fuerzas rivales: por un lado, los latifundistas y la Iglesia católica y, por otro, los terratenientes liberales y la burguesía comercial. Los primeros defendían a la clase semifeudal y los segundos representaban a la burguesía agraria, comercial e industrial. Estos partidos fueron denominados "históricos".

En Colombia, en 1848 se fundó el Partido Conservador y un año después surgió el Partido Liberal, cuya rivalidad suscitó guerras civiles y golpes de Estado. Una década más tarde, las revueltas populares iniciadas en abril de 1948 en respuesta al asesinato de Jorge Eliécer Gaitán, líder de izquierda del Partido Liberal, aceleraron el contacto entre los dos partidos, los cuales adoptaron una enmienda constitucional en 1957 que prescribía la sucesión alternada de ambas colectividades en la Presidencia hasta 1974; a ello se le llamó Frente Nacional. Lo mismo ocurrió en Honduras, en donde el Partido Nacionalista (conservador), fundado en 1891, rivalizaba con el Partido Liberal, surgido en el mismo año como organización de la burguesía media y pequeña.

La etapa siguiente de la historia de los partidos latinoamericanos se entronca en la Revolución cubana ${ }^{1}$ y el nuevo auge de la lucha de liberación nacional en los países del continente. Además de intensificar la polarización de las fuerzas políticas nacionales, esa Revolución contribuyó al surgimiento de partidos políticos con tendencia de izquierda.

En el siglo XIX encontramos como los partidos políticos más influyentes en toda América Latina el Partido Conservador colombiano, el Partido Conservador Ecuatoriano (PCE, desde 1861), el Partido Nacionalista de Honduras, el Partido Conservador Tradicionalista de Nicaragua (fundado en la primera mitad del siglo XIX) y la Asociación Nacional República de Paraguay (creada en 1881). En el sur del continente observamos partidos conservadores que defienden a la oligarquía terrateniente: en Argentina, el Partido Nacional Democrático Conservador, surgido después del golpe de Estado dirigido en 1930 por el general Uriburu y disuelto en 1943, cuando llegó al poder Juan Domingo Perón; sin embargo, después de derrocado

\footnotetext{
Se llevó a cabo en el año 1959. Provocó la caída de la dictadura del general Fulgencio Batista, para instaurar el nuevo régimen del líder Fidel Castro.
} 
el Gobierno peronista, se formaron dos partidos conservadores: el Democrático, con sede en la provincia de Córdoba y el Democrático Popular, en la Provincia de Buenos Aires. Tiempo después, en 1959, con el fin de reforzar sus posiciones, los conservadores se unieron en la Federación Nacional de Partidos del Centro.

Lo anterior evidencia que el "comienzo" de los partidos políticos en la mayoría de países de la región latinoamericana tienen en común el bipartidismo, en muchos casos conformados inconvenientemente por ideales contrapuestos que devienen de intereses personales.

En el presente escrito se tendrá como enfoque el mal llamado "multipartidismo", para tratar de dilucidar por qué la partidocracia se ha originado en torno a intereses personales de los líderes políticos que aprovechan temas de interés general desviados para generar asombro en los grupos sociales.

Empezaremos por conocer el surgimiento de los partidos políticos en Colombia, centrados en los conflictos ente las facciones políticas existentes desde la época de la Nueva Granada, anterior a la Gran Colombia.

Más adelante haremos una aproximación al surgimiento de las Constituciones Políticas, con las que se quiso dar una dirección democratizadora a las organizaciones políticas.

\section{El comienzo de los partidos políticos en Colombia}

La época de la conquista, iniciada desde el avistamiento de la península del departamento hoy conocido como La Guajira por parte de las expediciones enviadas por Colón, tuvo lugar en el año 1500. Más tarde, en 1526, Santa Marta fue fundada como ciudad española, de donde en 1536 partió una expedición encargada de conquistar a los muiscas y fue comandada por Gonzalo Jiménez de Quesada, comisionado por la corona española para explorar el nacimiento del río Magdalena. Al mismo tiempo partieron las expediciones de Sebastián de Belalcázar² y Nicolás de Federmán. ${ }^{3}$

Expedicionario español que fundó Popayán y Cali.

Expedicionario alemán a quien se le adjudicó la administración de la provincia de Venezuela. 
Por su parte, Gonzalo Jiménez de Quesada fundó Santa Fe (hoy Bogotá) y la nombró capital de todo el territorio conquistado, llamado en ese entonces Nueva Granada.

Desde esa época se crearon unas agrupaciones sociales que recibieron el nombre de facciones, con similitud práctica con el concepto de partido político. Facción viene de la palabra factio, proveniente del latín que significa "grupo de personas unidas por ideas o intereses comunes dentro de una agrupación o colectividad". ${ }^{4}$ Por otra parte, la noción de partido político es "conjunto de hombres unidos para promover, mediante su labor conjunta, el interés nacional sobre la base de algún principio particular". ${ }^{5}$ De lo anterior se puede concluir que las facciones son organizaciones antiguas de las cuales provienen los partidos políticos.

\subsection{Instituciones de la Nueva Granada}

En la segunda mitad del siglo XVI, España estableció el sistema definitivo de gobierno para la Nueva Granada, que consistió en una estructura centralizada y organizada jerárquicamente, constituida por:

1. El Consejo de Indias: el territorio era gobernado por el rey y los consejeros desde España. Era el órgano consultivo más importante, cuyos miembros servían simultáneamente como tribunal administrativo, órgano legislativo y corte de apelación.

2. Virreinato: era la más alta autoridad en el territorio americano, conformada por virreyes que disponían de una audiencia con funciones similares a las del Consejo de Indias. En 1717, la Capitanía General fue elevada a la categoría de Virreinato, al que se adscribieron las Presidencias de Quito y Panamá.

3. Capitanías Generales: eran la máxima autoridad en territorios lejanos al Virreinato y tenían poderes militares.

4. Presidencias: tenían la misma función de las Capitanías Generales, pero carecían de facultades militares.

Real Academia Española, "Facción" en Diccionario de la Real Academia Española, http://dle.rae.es/:id=HS2Wg2F (acceso noviembre 7, 2017).

5 Felipe Reyes Romo, "Facción y partido. Indagaciones sobre la historia de los conceptos", http://meme. phpwebhosting.com/ migracion/rimd/documentos_miembros/11700Facci\%F3n\%20y\%20Partido.pdf (acceso noviembre 7, 2017). 
Las instancias de poder (Virreinatos, Capitanías y Presidencias) estaban subdivididas territorialmente en provincias, cada una con su respectivo gobernador.

5. Cabildos: si bien es cierto que sus miembros eran escogidos de forma no democrática, en algunos casos se escogían residentes locales; por consiguiente, era la única institución colonial que tenía cierto carácter representativo. Podemos entender que esta institución de gobierno marca el inicio de lo que serían los partidos políticos, toda vez que su función principal era definir las condiciones de vida locales (bajo la presión del dinero o de las influencias) en favor de los habitantes coloniales.

\subsection{Impulsores de la Independencia}

\subsubsection{Rebelión de los Comuneros}

Como primer antecedente de la Independencia encontramos la facción de los Comuneros, la cual se inició como protesta contra el alza de los impuestos que España había implementado para costear su participación en la independencia de Estados Unidos ante Inglaterra. Se decretó que en la Nueva Granada era necesario el sostenimiento de la gran base naval en Cartagena, para lo cual tanto el monopolio gubernamental del tabaco como el del aguardiente subieron sus precios, lo que provocó la quema de tabaco y el derrame de aguardiente. En la provincia de Socorro, los habitantes se organizaron en una asamblea popular y eligieron como sus líderes a cinco criollos locales que ostentaron el título de capitanes generales; el más importante de ellos fue Juan Francisco Berbeo. Este fenómeno se repitió en varias provincias cercanas, en donde los habitantes, después de organizarse, elegían sus líderes como capitanes.

Una vez consolidadas las comunas, se suspendieron las protestas y se inició la comercialización, con el fin de recolectar recursos para su causa, obtener armas y deponer funcionarios públicos populares para posteriormente emprender la marcha hacia Santa Fe. Luego de una negociación con el arzobispo Antonio Caballero y Góngora, encargado por la audiencia de Santa Fe, terminó el levantamiento y se obtuvo la eliminación de los decretos fiscales. Por esta circunstancia se discute sobre si en realidad las facciones de los Comuneros configuran una rebelión por la independencia, pues lo que pretendían era la eliminación de los tributos; no obstante, hay quienes coinciden en que sí son un antecedente de los partidos políticos. 


\subsubsection{Antonio Nariño}

El fin de la rebelión no significó el término de las inconformidades, ya que cada vez aparecían más manifestaciones que involucraban a gente prestante de la sociedad criolla, como es el caso de Antonio Nariño, quien pertenecía a la alta clase de la sociedad de Santa Fe. Esta situación no le impidió tener cierta simpatía con las causas de los Comuneros y en particular con la causa de la Independencia. A finales de 1793 recibió de manos de un capitán de la guardia del palacio del virrey una copia del documento básico de la Revolución francesa y la Declaración de los derechos del hombre, textos que tradujo para difundirlos por toda la provincia; por ello, fue expulsado y encarcelado en África. Luego de escapar, se enfocó definitivamente en la Independencia. Tiempo después se entregó al nuevo virrey en 1797 y fue condenado a seis años de prisión. Cuando fue liberado, puso en marcha el movimiento independentista, posicionado como primer precursor de dicho movimiento y de la primera facción consolidada en la historia de la Nueva Granada, llamada independentista.

\subsubsection{Sucesos importantes para la conformación de los partidos políticos tradicionales}

Las autoridades instituidas en América juraron lealtad al rey legítimo José I impuesto por Napoleón, quien derrocó temporalmente al rey Fernando VII, pero los españoles nacidos en América Latina y los "criollos" expresaron su derecho a manejar las colonias durante la ausencia del rey. Por eso, se movilizaron en masa para la creación de juntas americanas que se consolidaron en 1810. Esto resume la disputa de las dos principales facciones por el poder de las colonias americanas: los independentistas, urgidos por su deseo de independencia ante España, querían su propio gobierno y los centralistas, quienes pretendían que las autoridades españolas continuaran ejerciendo el gobierno hasta que se derrocara al rey nombrado por Napoleón y se terminara la lucha con las invasiones francesas en España.

Paralelo a esto se crearon diversas juntas, incluida la Junta de la Capital como la más importante; sin embargo, los desacuerdos entre ellas, en razón a que cada uno quería tomar el control sobre el territorio, desataron los primeros intentos de organización política, período denominado Patria Boba (1810-1816).

Cartagena era la provincia de cabecera más interesada en independizarse de las juntas de Bogotá, pero no obtuvo el apoyo de su segunda provincia: Mompox, declarada 
"cabeza de una nueva provincia independiente con su propia junta". ${ }^{6}$ Fue en la disputa por el poder cuando se produjo el primer derramamiento de sangre en la Nueva Granada, en $1811 .^{7}$

Tras la libertad de Cartagena, Antonio Nariño siguió su revolución independentista, se posesionó como presidente de Cundinamarca y lideró la facción denominada patriota en su disputa con los realistas, facción conformada por el régimen español (centralista).

Antonio Nariño provocó el inicio de la guerra civil sostenida entre Bogotá y las Provincias Unidas de la Nueva Granada diferentes a los centralistas. Tuvo lugar entre 1812 y 1814, "cuando las tropas federales de las provincias unidas de la nueva granada conquistaron a Bogotá, con la ayuda del auxiliar venezolano Simón Bolívar", 8 justo en la ausencia de Antonio Nariño, quien se encontraba en la junta de Pasto luchando contra los realistas, fue apresado y enviado a España a cumplir su condena durante seis años.

Por lo anterior, Simón Bolívar fue el encargado de continuar con la lucha por una forma de gobierno independiente y declaró la independencia ante el régimen español en un suceso de hechos iniciado el 20 de julio de 1810, fecha en la que se instauró la primera junta de Santa Fe de Bogotá (día de la independencia de la actual Colombia).

La restitución del trono al rey Fernando VII en 1814 propició una respuesta masiva a la rebelión de las colonias de la Nueva Granada (encabezadas por Simón Bolívar), lo cual terminó con la alianza de los líderes José Antonio Páez, Francisco de Paula Santander y Simón Bolívar para conseguir la victoria final el 7 de agosto de 1819, en la batalla del puente de Boyacá.

En 1819, en el Congreso de Angostura se diseñó una nueva Administración, con Santander como vicepresidente y Bolívar como presidente, conocida como la Gran Colombia y conformada por las actuales Repúblicas de Colombia y Venezuela.

\footnotetext{
6 Germán Lozano Villegas, "Historia de los partidos políticos en Colombia", Via Inveniendi et Iudicandi, núm. 19 (2015): 11.

$7 \quad$ Lozano Villegas, "Historia de los partidos políticos en Colombia", 12.

8 Lozano Villegas, "Historia de los partidos políticos en Colombia", 13.
} 
No obstante, con el Congreso de Cúcuta, en 1821, se adoptó una forma de gobierno centralizada con sede en Bogotá, después de inevitables desacuerdos entre federalistas y centralistas.

La ruptura del sistema de gobierno planteado se debe a las protestas de Venezuela orientadas a exigir mayores beneficios, al considerar que la mayoría de los recursos que consumía la unión provenían de esa parte del territorio de la Gran Colombia. Se decidió la división de las dos Naciones, no solo por las protestas de los venezolanos, sino por las discordias existentes entre Bolívar y Santander: el primero consideró al vicepresidente como un derrochador de los recursos, por lo que se atribuyó el poder exclusivo al presidente.

Como consecuencia de la lucha de poderes, Santander y sus colaboradores impulsaron una campaña "en contra de Bolívar en el congreso y en la prensa", ${ }^{9}$ cuya facción se denominó santanderista y la de Bolívar, bolivariana. Así las cosas, los santanderistas conformaron el inicio del Partido Liberal (registrado en 1849) y los bolivarianos marcaron el origen del Partido Conservador (registrado en 1848).

En los años siguientes hubo una multiplicidad de discordias entre los dos partidos, que finalizó con la muerte de Simón Bolívar en la ciudad de Santa Marta, en la quinta de San Pedro Alejandrino el 17 de diciembre de 1830.

En aras de una constitución más estable se eligió a Santander en 1833, quien en señal de venganza conspiró contra los bolivarianos, en busca de un régimen federal. Su movimiento se llamó progresista.

\section{La influencia del sistema de partidos políticos en la democracia}

En la actualidad, en Colombia y en muchos países latinoamericanos, los sistemas de partidos políticos se han convertido en instituciones programáticas y de mejor practicidad, pues han dejado de lado al bipartidismo tradicional para dar paso al sistema multipartidista, el cual ha soportado múltiples críticas por perseguir fines constitucionalmente ajenos. Sistema multipartidista se ha definido como:

9 Lozano Villegas, "Historia de los partidos políticos en Colombia", 19. 
[La] atomización de los partidos tradicionales — Liberal y Conservador - y la fragmentación de las minorías políticas (agrupaciones políticas, religiosas, étnicas y regionales), donde no se habla de partidos políticos sino de microempresas electorales, entendidas como empresas electorales que se disputan el campo de la representación política. ${ }^{10}$

Por causa de este sistema no podemos hablar de verdaderos partidos políticos sino de "microempresas electorales, en la medida que son empresas individuales que se disputan el campo de la representación política" ${ }^{11}$ Es un multipartidismo engañoso, pues estamos presenciando la inevitable lucha estratégica para disputar el poder y la contratación pública que año a año se adjudica a los mismos agentes económicos. Entonces, tenemos que muchos de los partidos actuales se identifican más con una facción, que tiene una estructura frágil, sin ideales y sin intereses generales, en lugar de identificarse con el concepto de fracción: "[...] organización, estabilidad, significación electoral, identidad propia en torno a un líder, pero mantienen una gran dependencia del partido". ${ }^{12}$

En la actualidad de muchos Estados Naciones se evidencia un sistema multipartidista; no obstante, la realidad indica que ha sido un "fraccionamiento de los partidos políticos"13 tradicionales, como lo hemos vivido desde el inicio de la historia de Colombia. Por esa razón, en nuestra era tenemos figuras como la prohibición de la doble militancia o figuras como el "transfuguismo". ${ }^{14}$

Los partidos políticos tradicionales —el Partido Liberal y el Partido Conservadorhan sufrido una reducción considerable de sus miembros, especialmente a partir de la reforma de 2002. Esta abrió un espacio de competencia que, en principio, se pretendió "sana" o con procedimientos menos clientelistas.

De acuerdo con lo anotado, el primer impulso que mueve las iniciativas legislativas, políticas públicas, etc. es la guerra de poderes en el sistema "multipartidista" que

10 Eduardo Pizarro Leongómez, "Colombia: ¿Renovación o colapso del sistema de partidos?" en Colombia ante los retos del siglo XXI: desarrollo, democracia y paz, coord. Juan Ibeas Miguel y Manuel Alcántara Sáez (Salamanca: Universidad de Salamanca, 2001), 36.

11 Lozano Villegas, "Historia de los partidos políticos en Colombia", 129.

12 Lozano Villegas, "Historia de los partidos políticos en Colombia", 129.

13 Fernando Giraldo y Patricia Muñoz, Partidos politicos en Colombia: evolución y prospectiva (Bogotá: Fundación Konrad Adenauer, 2014), 44.

14 Enoc Francisco Morán Torres, "Transfuguismo: dicotomía entre la lealtad partidaria y la participación política. Una visión desde los precedentes judiciales en México", Díkaion 26, núm. 1 (2017): 89. 
tenemos, lo que ha influido en temas muy importantes para el país, como lo fue el Acuerdo final para la terminación del conflicto con el grupo ilegal FARC-EP. Es un asunto histórico para la Nación, que ha tenido serios inconvenientes desde sus cimientos, pero que sin duda alguna necesita del apoyo de todos los ciudadanos para seguir ejecutándose.

Si bien Colombia parte de ser una nación "democrática y participativa", ${ }^{15}$ el sistema democrático actual es incoherente por el fraccionamiento de los partidos políticos e inconveniente por no garantizar la participación de todos los sectores sociales; de tal forma, se presenta una "dicotomía entre la lealtad partidaria y la participación política". ${ }^{16}$

Lo anterior es el resultado de la creación de asociaciones políticas originadas por causas circunstanciales o personales, como es el caso del Centro Democrático, Partido de la Unidad Nacional, Cambio Radical y otros tantos creados en la última década del país.

Aunado a la proliferación de partidos políticos, muchas ideologías de partidos sin mayor número de miembros tienen una postura moderada y colaborativa con el Gobierno de turno, comparta o no sus ideales constitutivos. Aun así, estas organizaciones son presididas por dirigentes que adoptan posturas críticas, pero solo ante la opinión pública.

Por esta razón, no tenemos multipartidismo ni democracia representativa, pues solo basta ver que en el período de las elecciones tanto los miembros de los partidos como los ciudadanos nos comportamos de una manera grotesca contra los valores y la ética ciudadana.

\section{La previsión constitucional frente al sistema de partidos políticos en Colombia}

El sentido de las normas y los principios constitucionales era claro en el momento de diseñar el modelo de organizaciones políticas en los debates de la Asamblea Nacional Constituyente en Colombia en el año 1991. Este no era otro que el pluralismo político, de forma que se incluyeran todos los sectores sociales y se terminara la

\footnotetext{
República de Colombia, Constitución Política (Bogotá: Legis, 1991), preámbulo.

16 Morán Torres, "Transfuguismo: dicotomía entre la lealtad partidaria y la participación política", 96.
} 
partición electoral entre los dos partidos políticos tradicionales; además, se ideó el modelo de movimientos políticos y grupos de personas con fines políticos, como garantía de la inclusión de los ciudadanos a la democracia en el país. Asimismo, se amplió el derecho a elegir todos los cargos públicos representativos, en particular con la elección popular de los gobernadores de los departamentos. ${ }^{17}$

Con proyectos dirigidos al fortalecimiento de la democracia representativa y participativa, es decir, a la ampliación del derecho de elegir y ser elegido, se previó:

[El] reconocimiento constitucional de los partidos y movimientos políticos -Rama electoral independiente y autonomía-, establecimiento de la tarjeta electoral en todos los comicios; propuesta sobre la consagración del voto obligatorio; propuesta de elección popular; y elevación a la categoría constitucional del derecho a la oposición política y sus garantías. ${ }^{18}$

Así las cosas, en la Constitución Nacional se previó la creación de los partidos políticos por los ciudadanos, ${ }^{19}$ como muestra del derecho a la participación política y de libertad de reunión, ${ }^{20}$ de manifestación, ${ }^{21}$ de expresión, de asociación ${ }^{22}$ y de pensamiento.

Además, la Constitución Política de 1991 exige "organización democrática"23 y obliga a todas las organizaciones políticas y a sus miembros el cumplimiento de principios como el de "transparencia, objetividad, moralidad, la equidad de género, y el deber de presentar y divulgar sus programas políticos". ${ }^{24}$

17 Floralba Padrón Pardo, "Las organizaciones políticas en Colombia: reconocimiento constitucional y proceso de fortalecimiento" (tesis de pregrado, Universidad Externado de Colombia, 2012), 10.

18 Padrón Pardo, "Las organizaciones políticas en Colombia", 298.

19 "Todo ciudadano tiene derecho a participar en la conformación, ejercicio y control del poder político [...]". República de Colombia, Constitución Política, art. 40. "El Consejo Nacional Electoral reconocerá Personería Jurídica en la conformación, ejercicio y control del poder político grupos significativos de ciudadanos [...]". República de Colombia, Constitución Política, art. 108.

20 "Toda parte del pueblo puede reunirse y manifestarse pública y pacíficamente. Sólo la ley podrá establecer de manera expresa los casos en los cuales se podrá limitar el ejercicio de este derecho". República de Colombia, Constitución Política, art. 37.

21 "Se garantiza a toda persona la libertad de expresar y difundir su pensamiento y opiniones, la de informar y recibir información veraz e imparcial, y la de fundar medios masivos de comunicación". República de Colombia, Constitución Política, art. 20.

22 "Se garantiza el derecho de libre asociación para el desarrollo de las distintas actividades que las personas realizan en sociedad". República de Colombia, Constitución Política, art. 38.

23 Lozano Villegas, "Historia de los partidos políticos en Colombia", 121.

24 República de Colombia, Constitución Política, art. 107, num. 3. 
Lo que la Carta Superior previó fue que las organizaciones políticas garantizaran la participación y "representación democrática", ${ }^{25}$ es decir, la democracia en todas sus versiones, pues en este momento histórico no es dable pensar en una democracia directa como la que muy bien aplicaban en Roma en el período de la Primera República (509 a. C.-267 a. C.).

\section{La democracia en la justicia transicional del Acuerdo de paz}

Todo el esfuerzo del país con ocasión de la negociación y firma del Acuerdo final para la terminación del conflicto tiene como punto álgido la conformación de un tribunal de justicia transicional contentivo de mecanismos de carácter judicial y extrajudicial.

El 26 de agosto de 2012, el Gobierno nacional y el grupo armado ilegal FARC-EP suscribieron el Acuerdo final, que concluyó el 15 de diciembre de 2015 con la publicación de las conclusiones a las que llegaron las dos partes negociadoras.

Para hacer un análisis constructivo del Acuerdo, debemos partir de que hay muchos aspectos imposibles de incluir en un solo documento. Por tal motivo, se han revisado diversos problemas jurídicos derivados de su firma ${ }^{26}$ que pueden resumirse en:

1. Resolución de conflictos de competencia entre la jurisdicción especial para la paz y la jurisdicción ordinaria en todas sus especialidades.

2. La viabilidad de que los procesados en la jurisdicción especial para la paz acudan a mecanismos de tutela constitucionales al ver infringidos sus derechos.

3. La continuidad o absorción de los procesos iniciados con anterioridad a la firma del Acuerdo a los indiciados que se regirán por la justicia transicional para la paz.

25 Germán Lozano Villegas, "Los partidos políticos en Colombia: marco jurídico de creación, registro, funcionamiento y extinción”, Revista de Derecho Estasiológico. Ideología y Militancia 2, núm. 3 (2014): 106.

26 Para la doctora Caldas Botero, existen varios aspectos jurisdiccionales que no tuvieron lugar en las regulaciones del marco del Acuerdo general para la terminación del conflicto con el grupo ilegal FARC-EP. Luisa Fernanda Caldas Botero, "Aproximación a los problemas fundamentales de la justicia transicional. Especial énfasis en las sanciones imponibles en el marco de la jurisdicción especial para la paz", Derecho Penal y Criminología 37, núm. 102 (2016): 210. 
4. Sobre el supuesto de que los organismos de la justicia transicional para la paz pertenezcan a la Rama Judicial del poder público, no se previeron los mecanismos de solución de conflictos con las demás ramas del poder público.

5. La modificación e interpretación del Acuerdo final es un suceso casi inevitable en los períodos presidenciales futuros; no obstante, no se previeron mecanismos para ello.

Ante estos vacíos del Acuerdo, los programas políticos para las elecciones 2018-2022 han sido indiferentes frente a las preocupaciones superficiales de los líderes de los partidos políticos; en su lugar, tendrán como objetivo principal la modificación de los beneficios para los procesados que pertenecieron al grupo ilegal FARC-EP. Así, observaremos una clase política que manipula "los asuntos de guerra y paz en función de intereses electorales", ${ }^{27}$ los partidos políticos de extrema derecha encabezados por Álvaro Uribe apostarán por difundir el inexistente resentimiento generalizado en contra del Acuerdo final de terminación del conflicto armado.

\section{Conclusiones}

El declive de la democracia en nuestros tiempos se debe, en gran medida, a ciertos términos que hemos adoptado, como partidocracia o multipartidismo. Por partidocracia entendemos la concentración de poder en manos de los partidos políticos en detrimento de la democracia parlamentaria.

Como hemos dicho, los programas de gobierno de un partido político tienen origen en la voluntad particular de su líder, quien además propone el candidato presidencial — cuando no se autopostula — y ejercerá su correspondiente influencia para elección de Congreso y para nombramiento del Poder Judicial.

Por esta razón, dichas organizaciones políticas solo se llenan de contenido en los momentos electorales, pero es un contenido de poder y no ideológico, pues en este momento solo se formulan programas para traer la atención del público y se dejan por fuera los intereses constitucionales.

27 Marco Palacios, "En Colombia hay una guerra verdadera y muchas paces artificiales", Revista de Economía Institucional 18, núm. 35 (2016):340. 
Todos los programas políticos nacen dominados por la voluntad particular del líder del partido, lo cual se debe a que estas organizaciones pasaron de concebirse como un "conjunto de hombres unidos para promover, mediante labor conjunta, el interés nacional sobre la base de algún principio particular"28 a un conjunto de personas ligadas para satisfacer la voluntad de su dirigente político, sin considerar ideología o fin impresos en su creación.

De esta forma se genera el denominado "consacionalismo" en un Estado, término que pretende explicar los mecanismos de estabilidad política en sociedades con profundas divisiones. En otras palabras, es el medio con el cual se pretende lograr que el poder tenga una característica de unidad y autonomía y se tomen decisiones de acuerdo con todas las materias de interés general y respeto a la elección independiente en el resto de sectores. De conformidad con lo anterior, estos mecanismos de estabilidad solo pueden ser aplicados en sistemas políticos divididos, como son los de la región latinoamericana.

Por esto, es una tendencia generalizada creer que la democracia es un modelo de gobierno con mayúsculas falencias, pues las decisiones sobre políticas públicas no provienen del pueblo que, en un concepto romano, era el generador del poder de los dirigentes.

\section{Referencias}

Caldas Botero, Luisa Fernanda. "Aproximación a los problemas fundamentales de la justicia transicional. Especial énfasis en las sanciones imponibles en el marco de la jurisdicción especial para la paz". Derecho Penal y Criminología 37, núm. 102 (2016): 105-120.

Giraldo, Fernando y Patricia Muñoz. Partidos políticos en Colombia: evolución y prospectiva. Bogotá: Fundación Konrad Adenauer, 2014.

Lozano Villegas, Germán. "Los partidos políticos en Colombia: marco jurídico de creación, registro, funcionamiento y extinción". Revista de Derecho Estasiológico. Ideología y Militancia 2, núm. 3 (2014): 99-118.

Lozano Villegas, Germán. "Historia de los partidos políticos en Colombia". Via Inveniendi et Iudicandi, núm. 19 (2015): 11-42.

Morán Torres, Enoc Francisco. "Transfuguismo: dicotomía entre la lealtad partidaria y la participación política. Una visión desde los precedentes judiciales en México”. Díkaion 26, núm. 1 (2017): 84-106.

28 Reyes Romo, "Facción y partido", 10. 
Padrón Pardo, Floralba. "Las organizaciones políticas en Colombia: reconocimiento constitucional y proceso de fortalecimiento". Tesis de pregrado, Universidad Externado de Colombia, 2012.

Palacios, Marco. "En Colombia hay una guerra verdadera y muchas paces artificiales". Revista de Economía Institucional 18, núm. 35 (2016): 339-342.

Pizarro Leongómez, Eduardo. "Colombia: ¿Renovación o colapso del sistema de partidos?" en Colombia ante los retos del siglo XXI: desarrollo, democracia y paz, coordinado por Juan Ibeas Miguel y Manuel Alcántara Sáez, 99-126. Salamanca: Universidad de Salamanca, 2001.

Real Academia Española. "Facción" en Diccionario de la Real Academia Española. http://dle. rae.es/?id=HS2Wg2F (acceso noviembre 7, 2017).

República de Colombia. Constitución Politica. Bogotá: Legis, 1991.

Reyes Romo, Felipe. "Facción y partido. Indagaciones sobre la historia de los conceptos". http://meme.phpwebhosting.com/ migracion/rimd/documentos_ miembros/11700Facci\%F3n\%20y\%20Partido.pdf (acceso noviembre 7, 2017). 